ALICE-PUB-2001-09

5 April 2001

\title{
Heavy Ions at the LHC: Physics Perspectives and Experimental Program
}

\author{
J. Schukraft ${ }^{\mathrm{a}}$
}

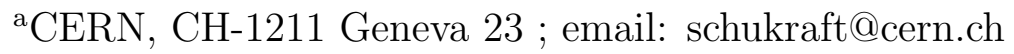

Ultrarelativistic heavy ion physics is entering the new era of collider experiments with the start-up of RHIC at BNL and construction for detectors at LHC well under way. At this crossroads, the article will give a summary of the experimental program and our current view of heavy ion physics at the LHC, concentrating in particular on physics topics that are different or unique compared to current facilities.

\section{Introduction}

The year 2000 was an extremely important and fruitful one for ultra-relativistic heavy ion physics. It started of with an appraisal of the CERN SPS Pb beam results [1] which concluded that 'compelling evidence has been found for a new state of matter' featuring many of the characteristics expected for a Quark-Gluon Plasma (QGP). Later that year the RHIC collider and its four experiments at BNL began operation with $\mathrm{Au}-\mathrm{Au}$ collisions at up to $130 \mathrm{GeV} /$ nucleon. Already this first short run was a success beyond even the most optimistic expectations, with physics results appearing in print and essentially flooding the relevant conferences within months. At this moment it is therefore difficult to shift the focus from the exciting present to the distant future, when around 2006 heavy ions will become available at a centre-of-mass energy of $5.5 \mathrm{TeV} /$ nucleon in the LHC. However, with RHIC in operation and construction for the LHC detectors well under way, it is both timely and important to ask what, if anything, the LHC heavy ion program can contribute beyond existing facilities to the study of strongly interacting matter under extreme conditions.

\section{Initial conditions and global event features}

In order to get some qualitative feeling about the changes in global event features to be expected when going up in energy by over two orders of magnitude from SPS via RHIC to LHC, some of the relevant parameters and predictions are listed in Table 1 and described below:

In elementary proton-proton reactions, both the charged particle multiplicity $d N_{c h} / d y$ [2] and the average transverse momentum $\left\langle p_{t}\right\rangle[3]$ grow only slowly (logarithmically) with $\sqrt{s}$. This would lead only to a modest growth in $d N_{c h} / d y$ (SPS:RHIC:LHC $=$ 0.75:1:2.1), $<p_{t}>(0.92: 1: 1.35)$ and energy density (roughly the product $d N_{c h} / d y \cdot p_{t}$ ).

\footnotetext{
${ }^{*}$ Invited talks given at the Quark Matter Conference in Stony Brook, USA, Jan. 2001 and the Int.
} Symposium on Nuclear Physics, Mumbai, India, Dec. 18-22, 2000. 


\begin{tabular}{|c|c|c|c|c|c|c|c|}
\hline & SPS & RHIC & LHC & $\mathrm{Pb}-\mathrm{Pb}$ & SPS & RHIC & LHC \\
\hline$\sqrt{s} / A(\mathrm{GeV})$ & 17 & 200 & 5500 & $d N_{c h} / d y$ & 400 & 1000 & $3-8 \times 10^{3}$ \\
\hline $\operatorname{pp} d N_{c h} / d y$ & 1.8 & 2.4 & 5 & $\tau_{0}^{\mathrm{QGP}}(\mathrm{fm} / c)$ & 1 & 0.2 & 0.1 \\
\hline $\operatorname{pp} p_{t}(\mathrm{GeV})$ & 0.36 & 0.39 & 0.53 & $\varepsilon\left(\mathrm{GeV} / \mathrm{fm}^{3}\right)$ & 3 & 35 & 500 \\
\hline $\mathrm{AA} d N / d y$ & $\propto N_{\text {part }}$ & $?$ & $\propto N_{c o l}$ & $\tau_{\mathrm{QGP}}(\mathrm{fm} / c)$ & $\lesssim 2$ & $2-4$ & $\gtrsim 10$ \\
\hline partons in $\mathrm{p}$ & 4 & 10 & 30 & $\tau_{\mathrm{fo}}(\mathrm{fm} / c)$ & 10 & $20-30$ & $30-40$ \\
\hline shadowing & 1 & 0.8 & 0.5 & $V_{\mathrm{loc}}\left(\mathrm{fm}^{3}\right)$ & few $10^{3}$ & few $10^{4}$ & few $10^{5}$ \\
\hline$d N_{p} / d y[9]$ & & 1400 & 5100 & $d E_{t} / d y$ & $(\mathrm{GeV})$ & 2000 & 17000 \\
\hline$d N_{p} / d y[10]$ & & $80-120$ & $1.5-5 \times 10^{3}$ & $d E_{t} / d y$ & $(\mathrm{GeV})$ & $200-300$ & $5-15 \times 10^{3}$ \\
\hline
\end{tabular}

However, also the scaling when going from pp to central AA reactions changes drastically over this energy range. While at low cms energy particle production is, to a very good approximation, proportional to the number of participants $N_{\text {part }}$, i.e. scales like $A$ in central AA collisions, hard processes, which dominate at high energies, scale proportional to the number of collisions $N_{c o l}\left(\propto A^{4 / 3}\right)$. Compared to SPS, this leads to an additional enhancement factor for $d N / d y$ in central AA reactions of about three (assuming equal contributions of soft and hard processes) or six (assuming hard dominance) for RHIC and LHC, respectively. In addition, as structure functions rise strongly at small $\mathrm{x}$, the number of partons available for collisions increases a function of energy. As an illustration, the number of partons inside the proton above $2 \mathrm{GeV}$ is listed in the table as row 'partons p' [4]. While some of this increase is reduced by shadowing in heavy nuclei, the net effect for particle production, roughly proportional to the square of parton number times shadowing reduction, is still very sizeable: A heavy nucleus contains about twice (four times) as many effective partons at RHIC (LHC) than at the SPS. The fact that nuclei get 'denser' at high energy is a great bonus which was not anticipated only a few years ago!

The right hand side of Table 1 summarises a selection of different measurements and estimates for global event features in $\mathrm{Pb}-\mathrm{Pb}$ collisions (for a similar table, including some original references, see [5]). While $d N_{c h} / d y$ has been measured at the SPS and can be extrapolated with confidence from the current RHIC results [6] to full RHIC energy, the predictions for LHC vary wildly between some 3000 and up to 8000 charged particles per unit rapidity. The main reasons for this spread are connected e.g. with uncertainties in gluon shadowing, final state parton saturation [7] and jet-quenching [8], all of which are expected to be rather different at RHIC and LHC. Therefore we might not be able to narrow down significantly this large range before LHC start-up even if more detailed results will become available from RHIC. Both the quantum mechanically determined 'formation time' of harder partons $\left(\propto 1 / p_{t}\right)$ and the increased interaction rate at high parton density decrease the thermalization time $\tau_{0}^{\mathrm{QGP}}$ of the dense medium, leading to extreme energy densities $\left(\varepsilon\left(\tau_{0}\right) \propto 1 / \tau_{0}\right)$ and temperatures at very early times during the collision at the LHC. As the system has to expand and cool to freeze-out conditions which are probably similar at all energies, QGP lifetime $\tau_{\mathrm{QGP}}$, freeze-out time $\tau_{\text {fo }}$ and final (local) freeze-out volume $V_{\text {loc }}$ likewise increase substantially. In particular the strong 
increase in QGP lifetime, both in absolute terms, in units of relaxation times (which in general decrease in denser systems), as well as compared to the hadronic phase, might be particularly relevant as it should enhance the relative contribution of signals generated in and sensitive to the QGP phase.

Finally, the last two rows show two representative pQCD calculations of initial parton density $d N_{p} / d y$, dominantly gluons, and transverse energy production $d E_{t} / d y$ [9] [10] While they differ in detail in their treatment of shadowing, parton saturation effects and $p_{t}$ cut-off, they qualitatively agree that densities should increase by about an order of magnitude from RHIC to LHC, much stronger than the logarithmic rise seen in pp reactions.

In summary, by increasing the energy from soft to hard dominated regimes we gain independently on several fronts: More partons are available, their inelastic interactions get both more effective (already in pp) and in addition more frequent (in nuclei), and the thermalized high temperature phase is established more rapidly and lasts longer.

\section{Qualitative changes at LHC}

Besides better initial conditions, a number of qualitative changes are expected at high energy leading to a very different system and observables which are either new or can be studied with greater precision.

Parton saturation: At LHC, and to a smaller extend at RHIC, the density of low $-x$ partons both in the incoming nuclei and in the final state after scattering are so high that the phase space for low momentum partons comes close to saturation and gluon merging becomes important to an extend that the reactions are sometimes referred to as a collision between two 'gluon walls' [7]. In the initial state, structure functions are modified by shadowing, and also in the final state the gluon density is reduced, limiting in a self-consistent way the infrared divergence of pQCD cross sections. Due to the large occupation numbers, the scattering process might be described by classical field theory, a new regime that becomes accessible for the first time with heavy ions at LHC. This 'gluon saturation' is expected to set in around $2 \mathrm{GeV}$ at LHC [9] where pQCD might be applicable and theoretical concepts can be tested.

Baryon density: The incoming nuclei are slowed down during the collisions and the net baryon number is distributed over typically 3-5 units in rapidity. This leads to a high baryon density at SPS and below, whereas it is not sufficient to populate the central region at $\mathrm{LHC}$, which will have a vanishing baryochemical potential $\mu_{b}$ comparable to the early universe. Therefore very different regions of the phase diagram will be explored at low energy ('compression') and high energy ('heating'). At RHIC, first indications are that a baryon excess persists, which is however small and probably not very relevant $\left(\mu_{b} / T \ll 1\right)$.

Energy density: Energy density and pressure, as calculated on the lattice, are shown in Figure 1, together with the estimated temperature range accessible at different machines. The SPS is certainly very close to, and likely already reaches beyond the hadronic phase; RHIC is ideally suited to explore the phase boundary in great detail; but only LHC will reach deep into the QGP and could approach the Stefan-Boltzman limit of an 'ideal gas' of QCD quanta. The region between $T_{c}$ and the SB limit is of particular interest 


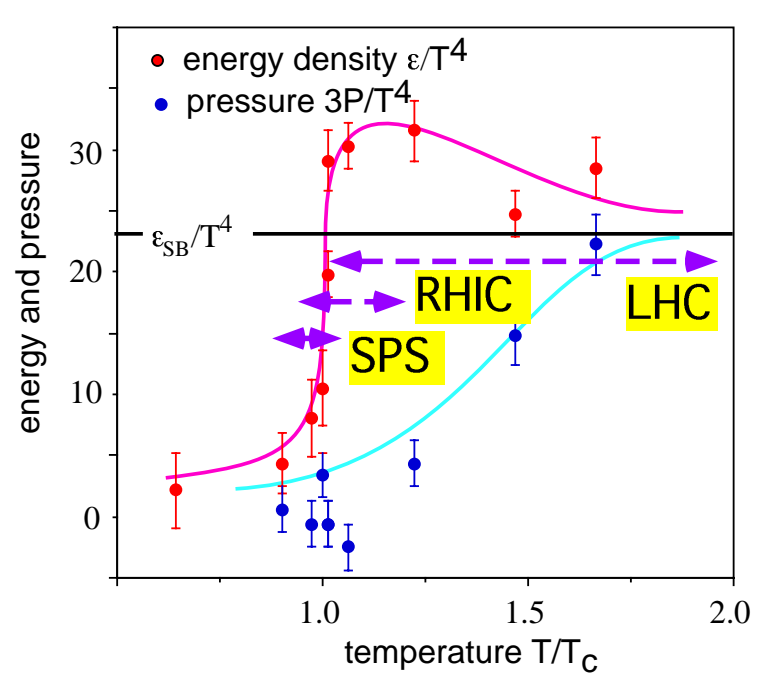

Figure 1. Energy density and pressure as calculated on the lattice as a function of temperature $\mathrm{T}$ in units of the critical temperature $\mathrm{T}_{c}$. The ideal gas StefanBoltzman limit is labeled $\varepsilon_{S B}$. The temperature range accessible at SPS, RHIC and LHC are indicated with arrows.

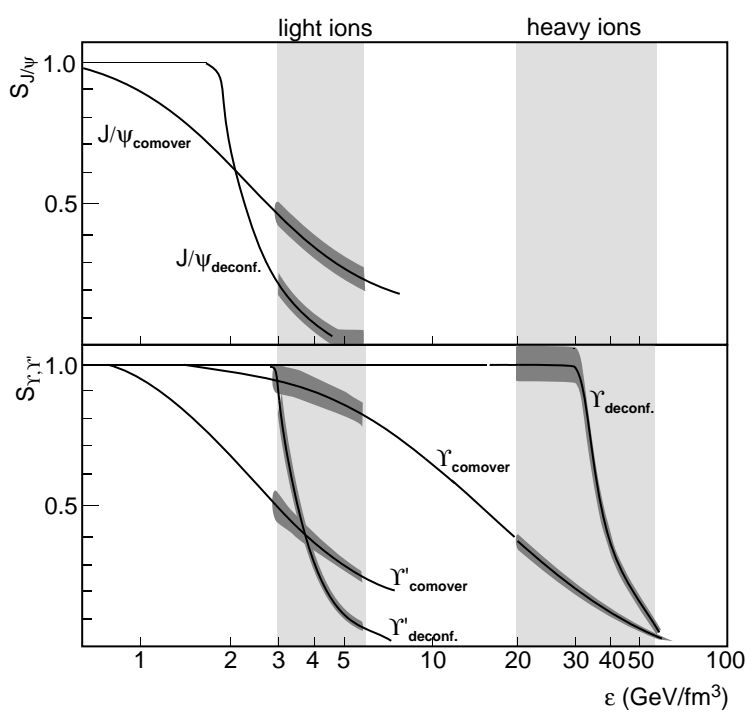

Figure 2. Energy density dependency of charmonium and bottonium survival probabilities as expected from melting in a deconfined medium and from the comover model. The precision of the measurement in ALICE after one month of running is shown as $1 \sigma$ error bands.

in order to study the interactions responsible for the deviation from the ideal parton gas case.

Hard processes: Hard scattering with large momentum transfer becomes significant at RHIC (measurable up to about $20 \mathrm{GeV}$ ) and copious at LHC (up to several hundred $\mathrm{GeV})[11]$. These hard probes, in particular high $p_{t}$ jets and heavy quark production, are formed at very early times and therefore will test the surrounding dense medium. The study of the classical deconfinement signal, i.e. J/ $\Psi$ suppression, can be extended at LHC to the Y family. A full 'spectral analysis' of heavy quarkonia states [12] will be much more difficult at RHIC, as the cross sections are lower by at least an order of magnitude [10] and the initial temperature is likely to be below the melting point of the tightly bound Y state (Figure 2). Open charm (and eventually beauty) production will be sufficiently frequent to add these heavy quarks into the list available for abundance analysis ('chemical analysis' of parton or hadron ratios, currently limited to up, down and strange quarks). This will help in disentangling the different stages - initial production, pre-equilibrium and thermal processes, hadronization - which are currently a major source of confusion in interpreting the measured ratios. 'Jet-quenching', the medium induced energy loss of hard partons [8], is a new probe not available at the SPS. It might be visible already in the shape of the inclusive hadron $p_{t}$ spectrum at RHIC, but should greatly improve in terms of clarity and theoretical tractability once absolute jet cross sections and fragmentation functions are measured at $\mathrm{LHC}$ in the $E_{t}$ range of tens to hundreds of $\mathrm{GeV}$. 
Thermal radiation: Observation of direct photons emitted from the hot initial reaction volume is a crucial piece of information which is currently still lacking or at least ambiguous. As the signal strength improves dramatically with energy density and plasma life-time, it might be observable at the colliders, in particular if the background from high $p_{t} \pi^{0}$ 's is reduced by thermalization and jet-quenching.

Event-by-Event physics: A unique feature of heavy ion collisions is the possibility to measure a number of observables on an even-by-event basis. Non-statistical fluctuations in these observables are of interest [13] because they are, in general, associated with critical phenomena in the vicinity of a phase transition, they can be related to thermal quantities (eg the heat capacity), or might be indicative of 'anomalous' events as suggested by cosmic ray observations (Centauro's etc.). Currently, with clear guidance and precise predictions from theory missing, this subject is essentially data driven. However, as the accuracy will in general increase inversely with the number of observed particles $(\propto 1 / \sqrt{N})$, EbE physics will become a precision instrument at high particle multiplicity and any observation of non-trivial fluctuating would be a clean 'smoking gun'. Even in the absence of anomalous fluctuations, some EbE measurements will be extremely useful to study the correlation with other inclusive observables, like the azimuthal dependence of jet-quenching with respect to the event plane which can be determined via the elliptic flow.

\section{Heavy ions in the $\mathrm{LHC}$}

The LHC will start to collide protons at $\sqrt{s}=14 T e V$ in early 2006 and will provide the first heavy ion collisions $(\mathrm{Pb}-\mathrm{Pb})$ towards the end of its first year of operation at a total cms energy of $1148 \mathrm{TeV}(\sqrt{s}=5.5 \mathrm{TeV}$ per nucleon for $\mathrm{Pb}-\mathrm{Pb})$. The sharing between proton and ion runs is expected to be similar to the one used in the past at the SPS, i.e. a long proton run $\left(10^{7} \mathrm{~s}\right.$ effective time) followed by a few weeks $\left(10^{6} \mathrm{~s}\right)$ of heavy ion or proton-nucleus collisions. The ion luminosity is limited for heavy systems by the short beam life-time and energy deposition in the supra-conducting machine magnets (quench protection), and for light ions by the injector chain. It reaches values between $3 \cdot 10^{31} \mathrm{~cm}^{-2} \mathrm{~s}^{-1}$ for light ions $\left(\mathrm{O}^{16}\right)$ and $10^{27} \mathrm{~cm}^{-2} \mathrm{~s}^{-1}$ for $\mathrm{Pb}$ ions. Proton- or deuteronnucleus collisions are feasible and foreseen, however only at equal magnetic rigidity per beam as the bending field is identical in both rings ('two-in-one' magnet design of the LHC). Further information on heavy ion operation of the LHC can be found in ref. [14].

\section{The dedicated heavy ion experiment ALICE}

ALICE is a general-purpose heavy-ion detector designed to study the physics of strongly interacting matter and the quark-gluon plasma in nucleus-nucleus collisions at the LHC. It currently includes more than 900 physicists - both from nuclear and high energy physics - from about 70 institutions in 25 countries.

The detector is designed to cope with the highest particle multiplicities anticipated for $\mathrm{Pb}-\mathrm{Pb}$ reactions $(\mathrm{dN} / \mathrm{dy} \approx 8000)$ and it will be operational at the start-up of the LHC. In addition to heavy systems, the ALICE Collaboration will study collisions of lowermass ions, which are a means of varying the energy density, and protons (both pp and p-nucleus), which provide reference data for the nucleus-nucleus collisions.

ALICE (Fig. 3) consists of a central part, which measures hadrons, electrons and pho- 
tons, and a forward spectrometer to measure muons. The central part, which covers polar angles from $45^{\circ}$ to $135^{\circ}$ over the full azimuth, is embedded in the large L3 solenoidal magnet. It consists of an inner tracking system (ITS) of high-resolution silicon tracking detectors, a cylindrical TPC, three particle identification arrays of time-of-flight (TOF), Ring Imaging Cerenkov (HMPID) and Transition Radiation (TRD) counters and a single-arm electromagnetic calorimeter (PHOS). The forward muon arm $\left(2^{\circ}-9^{\circ}\right)$ consists of a complex arrangement of absorbers, a large dipole magnet, and fourteen stations of tracking and triggering chambers. Several smaller detectors (ZDC, PMD, FMD, CASTOR, T0) are located at very forward angles.

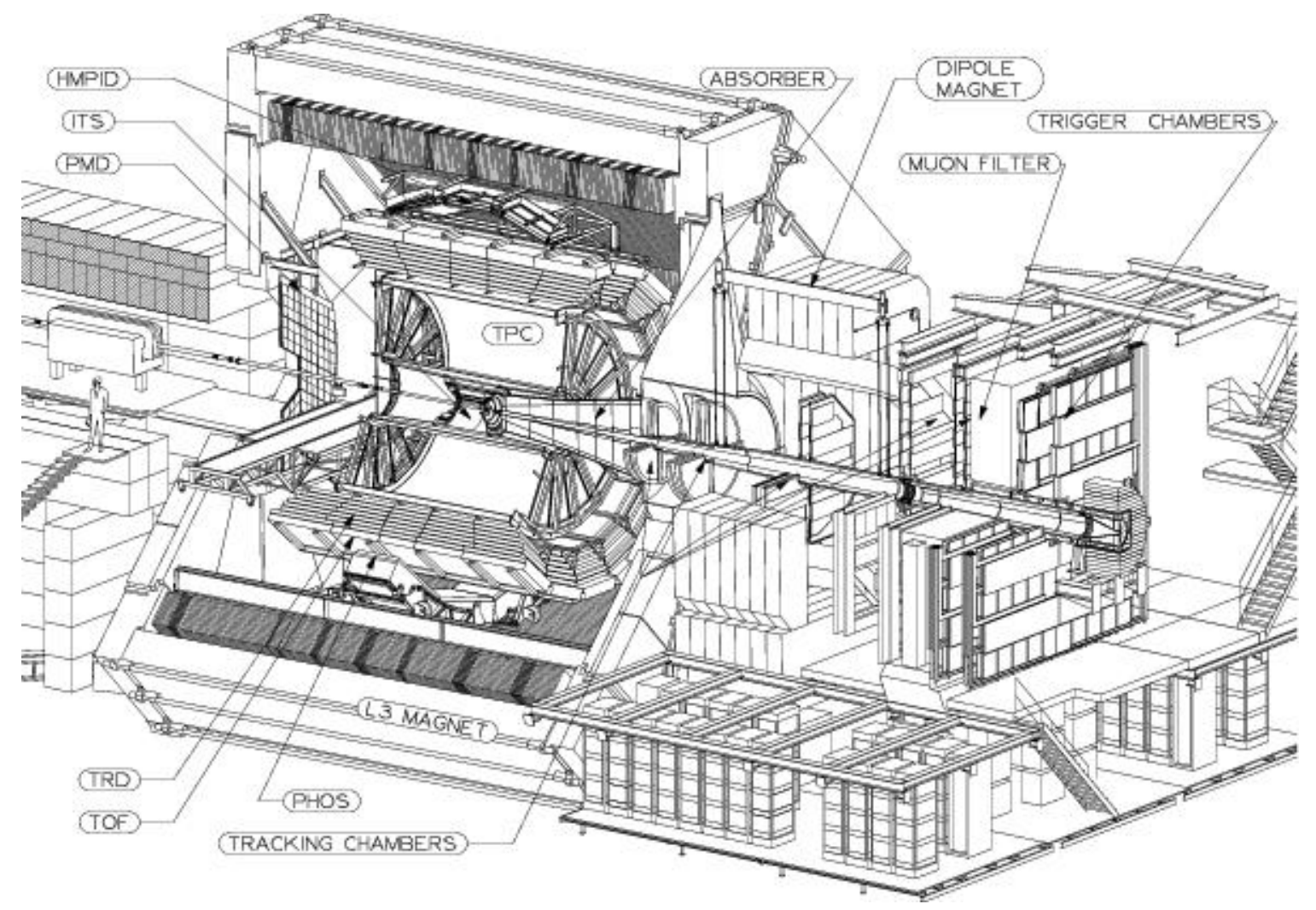

Figure 3. Artists view of the ALICE detector.

Magnet: The optimal choice for ALICE is a large solenoid with a weak field $(<0.5 \mathrm{~T})$ allowing full tracking and particle identification inside the magnet. The available space has to be sufficiently large to accommodate the PHOS, which must be placed at a distance of $\approx 5 \mathrm{~m}$ from the vertex, because of the large particle density. The magnet of the L3 experiment fulfils all these requirements.

Inner Tracking System: The basic functions of the inner tracker - secondary vertex reconstruction of hyperon and charm decays, particle identification and tracking of lowmomentum particles, and improvement of the momentum resolution - are achieved with six barrels of high-resolution detectors. Because of the high particle density, the innermost 
four layers need to be truly two-dimensional devices, i.e. silicon pixel and silicon drift detectors. The outer layers, at $\mathrm{r} \approx 50 \mathrm{~cm}$, will be equipped with double-sided silicon micro-strip detectors. Four of the layers will have analog readout for independent particle identification via $\mathrm{dE} / \mathrm{dx}$ in the non-relativistic region, which will give the inner tracking system a stand-alone capability as a low $\mathrm{p}_{t}$ particle spectrometer.

Time Projection Chamber: The need for efficient and robust tracking of up to 12000 charged particles within the acceptance has led to the choice of a TPC as the main tracking system. The inner radius of the TPC $(\mathrm{r} \approx 90 \mathrm{~cm})$ is given by the maximum acceptable hit density, the outer radius of $250 \mathrm{~cm}$ by the length required for a $\mathrm{dE} / \mathrm{dx}$ resolution of $<10 \%$. The design of the readout chambers and electronics, as well as the choice of the operating gas, is optimised for good double-track resolution.

Particle Identification System: Particle identification over a large part of the phase space and for many different particles is an important design feature of ALICE. A large Time-of-Flight array $\left(>100 \mathrm{~m}^{2}\right)$ at a radius of about $3.5 \mathrm{~m}$ is made with novel multigap resistive plate chambers (MRPC) with an intrinsic time resolution of $<100 \mathrm{ps}$. The proximity focusing RICH detector HMPID, of smaller acceptance and at larger radii, is optimised for the detection of high $p_{t}$ particles and will extend the accessible momentum range for inclusive particle spectra into the semi-hard region. The six layer Transition Radiation Detector (TRD) will identify electrons with momenta above $1 \mathrm{GeV} / \mathrm{c}$ to study quarkonia suppression and heavy quark production (charm, beauty) in the central acceptance.

Photon Spectrometer: The electromagnetic calorimeter will be located below the interaction region at $4.6 \mathrm{~m}$ from the vertex, and covers $8 \mathrm{~m}^{2}$ with $17 \mathrm{k}$ channels of scintillating $\mathrm{PbWO}_{4}$ crystals to measure direct photons and high $\mathrm{p}_{t}$ neutral mesons. These very dense crystals are needed to cope with the large particle density, and to have sufficient light-output to allow readout with silicon photodiodes.

Forward Muon Arm: The forward muon arm is designed in order to cover the complete spectrum of heavy quark resonances, i.e. , J/ $\Psi, \Psi^{\prime}, \mathrm{Y}, \mathrm{Y}^{\prime}, \mathrm{Y}^{\prime \prime}$ (Figure 2). It will measure the decay of these resonances, both in proton-proton and in heavy-ion collisions. The angular acceptance of the muon spectrometer is from $2^{\circ}$ to $9^{\circ}(\eta=2.5-4)$. Its mass resolution will be around $100 \mathrm{MeV}$ at $10 \mathrm{GeV}$, sufficient to separate all resonance states. It consists of a composite absorber, made with layers of both high- and low-Z materials, starting $90 \mathrm{~cm}$ from the vertex, a large dipole magnet with a $3 \mathrm{Tm}$ field integral placed outside the L3 magnet, and 10 planes of thin, high-granularity tracking stations (Cathode Strip and Cathode Pad Chambers). A second absorber at the end of the spectrometer and four more detector planes (RPC's) are used for muon identification and triggering. The spectrometer is shielded throughout its length by a dense absorber tube, of about 60 $\mathrm{cm}$ outer diameter, which surrounds the beam pipe.

Forward Detectors: ALICE uses a number of smaller detector systems (ZDC, PMD, FMD, CASTOR, T0) located at forward angles to define and trigger on global event characteristics. Four small and very dense calorimeters (Zero Degree Calorimeters, ZDC) are located about $100 \mathrm{~m}$ inside the machine tunnels on both sides of the interaction to define the impact parameter of the collision. A similar calorimeter (CASTOR), located closer to the interaction region on side of the experiment, will measure electromagnetic and hadronic transverse energy at large rapidity. The Forward Multiplicity Detector 
(FMD) measures charge particle production over a large fraction of phase space $(|\eta|<4)$. The Photon Multiplicity Detector (PMD) will search for non statistical fluctuations in the ratio of photons to charged particles, measure collective flow and transverse energy of neutral particles, and in addition determine the reaction plane. The T0 detector will measure the event time with great precision.

Trigger, Data acquisition and Offline: The ALICE trigger is foreseen to work on five levels, starting with a fast minimum bias interaction pre-trigger (issued after $<1 \mu \mathrm{s}$ ) to strobe some of the front-end electronics and ending with a high level online computing farm of several hundred PC's (L3 trigger) which is intended for further event selection (for example on high $\mathrm{p}_{t}$ jets or high mass lepton pairs) as well as online pre-tracking and event compression. Several detectors provide input to the different trigger levels to select e.g. for centrality, high $p_{t}$ electrons, muons, or photons.

The relatively short heavy-ion running period and the very large event sizes (up to 80 Mbyte even after zero suppression) determine the main features of the DAQ. In order to collect a sufficient number of events for physics analysis, the DAQ system has to be designed with a very large bandwidth up to 1.25 GByte/s on mass-storage. The DAQ architecture is based on a network of high-speed links linking all the data sources and the data destinations through a switch. This architecture provides the required flexibility and scalability to run in very different modes.

A new Off-line framework (ALIRoot) has been developed since 1998 based on all new $\mathrm{C}++$ code and the OO paradigm. The ROOT framework was adopted as a base for this development, integrating currently the GEANT 3 and later also the GEANT 4 simulation package. At the moment a complete OO simulation of ALICE exists and the OO reconstruction code is being developed in this framework.

\section{Conclusion}

Some 5 years from now, a new regime of very high energy density but low baryon density will be accessible with heavy ion collisions at the LHC. At some 30 times RHIC design energy, the step in energy from RHIC to LHC will be enormous, in fact larger than the one going from SPS to RHIC. LHC will reach, and even extend, the energy range probed by cosmic ray nucleus-nucleus collisions. Extrapolating from present results, all parameters relevant to the formation of the Quark-Gluon Plasma (QGP) will be more favourable: the energy density, the size and lifetime of the system, as well as relaxation times should all improve by a large factor, typically by an order of magnitude compared to SPS and even RICH. It should then be possible to obtain energy densities far above the deconfinement threshold, and to probe the QGP in its asymptotically free 'ideal gas' form. Unlike at lower energies, the central rapidity region will have a nearly vanishing baryon number density, similar to the state of the early universe. Reactions will be dominated in the early pre-equilibrium stage by a very dense system of semi-hard partons ('mini-jets'), which would lead to rapid thermalization and extremely high initial temperatures. Hard probes (heavy quark production, jets, even weak bosons) will be copiously produced well into the $100 \mathrm{GeV}$ mass and momentum range providing a new and, with pQCD, well calibrated tool to study the QGP.

The LHC will therefore complement, extend and eventually succeed the ongoing heavy 
ion program by providing a very different, and in many instances significantly better, environment for the study of strongly interacting matter.

With the past at AGS/SPS very productive and the present at RHIC extremely exciting, the field of ultra-relativistic heavy ion physics may confidently look forward to a promising future at LHC.

\section{REFERENCES}

1. U. Heinz and M. Jacob, preprint nucl-th/0002042.

2. F. Abe et al., Nucl. Phys A661 (1991) 690.

3. C. Albajar et al., Nucl. Phys B335 (1990) 261.

4. K.J. Eskola, private communication.

5. K. J. Eskola, International Europhysics Conference on High-energy Physics : EPSHEP '99, Tampere, Finland, 15 - 21 Jul 1999 - 1999, preprint hep-ph/9911350.

6. B. B. Back et al. [PHOBOS Collaboration], Phys. Rev. Lett. 85 (2000) 3100.

7. A.H. Mueller and J. Qiu, Nucl. Phys. B268 (1986) 427; L.D. McLerran and R. Venugopalan, Phys. Rev. D49 (1994) 3352. A.H. Mueller, Nucl. Phys. B572 (2000) 227; W. Pöschl and B. Müller, Phys. Rev. D60 (1999) 114505.

8. R. Baier, Y. L. Dokshitzer, A. H. Mueller, S. Peigne and D. Schiff, Nucl. Phys. B483 (1997) 291; M. Gyulassy, P. Levai and I. Vitev, Phys. Rev. Lett. 85 (2000) 5535; U.A. Wiedemann, Nucl. Phys. B588 (2000) 303.

9. K.J. Eskola, K. Kajantie, P.V. Ruuskanen, and K. Tuominen, Nucl. Phys. B570 (2000) 379 .

10. V. Emel'yanov, A. Khodinov, S.R. Klein, and R. Vogt, Phys. Rev. C 61 (2000) 044904.

11. X.N. Wang, Phys. Rep. 280 (1997) 287.

12. F. Karsch and H. Satz, Z. Phys. C 51 (1991) 209.

13. H. Heiselberg, subm to Phys. Rep., preprint nucl-th/0003046; M. Bleicher, S. Jeon and V. Koch, Phys. Rev. C 62 (2000) 061902; M. Asakawa, U. Heinz and B. Muller, Phys. Rev. Lett. 85 (2000) 2072.

14. D. Brandt CERN-LHC-Project-Report-450 ; CERN , 20 Dec 2000 http://documents.cern.ch/archive/electronic/cern/preprints/lhc/lhc-project-report450.pdf. 